\title{
Endoplasmic reticulum-associated degradation of glycoproteins in plants
}

\section{Silvia Hüttner and Richard Strasser *}

Department of Applied Genetics and Cell Biology, University of Natural Resources and Life Sciences, Vienna, Austria

\section{Edited by:}

Marisa Otegui, University of

Wisconsin at Madison, USA

\section{Reviewed by:}

Lorenzo Frigerio, University of

Warwick, UK

Federica Brandizzi, Michigan State

University, USA

\section{*Correspondence:}

Richard Strasser, Department of Applied Genetics and Cell Biology, University of Natural Resources and Life Sciences, Muthgasse 18, 1190

Vienna, Austria.

e-mail: richard.strasser@boku.ac.at
In all eukaryotes the endoplasmic reticulum (ER) has a central role in protein folding and maturation of secretory and membrane proteins. Upon translocation into the ER polypeptides are immediately subjected to folding and modifications involving the formation of disulfide bridges, assembly of subunits to multi-protein complexes, and glycosylation. During these processes incompletely folded, terminally misfolded, and unassembled proteins can accumulate which endanger the cellular homeostasis and subsequently the survival of cells and tissues. Consequently, organisms have developed a quality control system to cope with this problem and remove the unwanted protein load from the ER by a process collectively referred to as ER-associated degradation (ERAD) pathway. Recent studies in Arabidopsis have identified plant ERAD components involved in the degradation of aberrant proteins and evidence was provided for a specific role in abiotic stress tolerance. In this short review we discuss our current knowledge about this important cellular pathway.

Keywords: endoplasmic reticulum, protein degradation, protein quality control, ubiquitin-proteasome, protein glycosylation
Quality control and maintenance of protein homeostasis in the endoplasmic reticulum (ER) require the expression of molecular chaperones that assist protein folding and the recognition and elimination of non-native proteins by proteolytic degradation (Hegde and Ploegh, 2010; Smith et al., 2011). Many soluble and membrane-bound proteins are glycoproteins and the recognition of specific protein-bound sugar residues by lectins as well as the processing of the protein-bound oligosaccharides by glycosylation enzymes are crucial events during protein folding and ER-quality control processes (Helenius and Aebi, 2004). Nascent polypeptides that enter the ER are frequently glycosylated at asparagine residues in Asn-X-Ser/Thr consensus sequences. This type of protein glycosylation (termed $\mathrm{N}$-glycosylation) occurs co-translationally in the ER by transfer of the oligosaccharide precursor (Figure 1A). Immediately after the transfer the two terminal glucose residues are cleaved off by $\alpha$-glucosidase I and II and the resulting polypeptides with monoglucosylated glycan structures are subjected to the calnexin/calreticulin cycle (Figure 1B). In this quality control process, a soluble calreticulin or the membrane-anchored calnexin binds to the monoglucosylated oligosaccharide, promotes folding, and prevents aggregation of folding intermediates (Helenius and Aebi, 2004; Caramelo and Parodi, 2008). Properly folded proteins are released from this $N$-glycan-dependent quality control mechanism and can exit the ER to the Golgi. If, however, folding of glycoproteins is not successful, mannose residues are trimmed by $\alpha$-mannosidases, which generates a specific glycan signal that flags the glycoprotein for ER-associated degradation (ERAD) in yeast and mammals (Aebi et al., 2010; Hosokawa et al., 2010). ERAD

Abbreviations: ER, endoplasmic reticulum; ERAD, endoplasmic reticulumassociated degradation; MRH, mannose-6-phosphate receptor homology; UPR, unfolded protein response. is the main route for clearance of aberrantly folded proteins from the ER and is subdivided into distinct steps involving the recognition of the ERAD substrate, dislocation (retrotranslocation) to the cytosol, ubiquitylation, and finally the degradation by the $26 \mathrm{~S}$ proteasome.

\section{ERAD OF GLYCOPROTEINS IN YEAST AND MAMMALIAN CELLS}

In different eukaryotic cells, glycosylated and non-glycosylated ER-luminal as well as integral membrane proteins have been identified as ERAD substrates. Based on the location of the misfolded lesion in the protein that is subjected to disposal, $\mathrm{ERAD}_{\mathrm{L}}$ (lesion in the luminal region), $\operatorname{ERAD}_{\mathrm{C}}$ (defect in the cytoplasmic region), and ERAD ${ }_{M}$ (defect in transmembrane domain) substrates have been distinguished (Vashist and Ng, 2004; Bernasconi et al., 2010). Analysis of individual components of the ERAD complex and a recent systematic investigation of the ERAD interaction network and substrate-specific degradation pathways (Denic et al., 2006; Sato et al., 2009; Christianson et al., 2012) revealed that the yeast and mammalian ERAD complexes use a highly adaptive mechanism to remove different classes of substrate proteins. In yeast, the membrane-bound DOA10 E3 ubiquitin ligase complex is involved in degradation of $\mathrm{ERAD}_{\mathrm{C}}$ substrates, while the HRD complex is required for disposal of $E R A D_{L}$ and $E R A D_{M}$ substrates. The HRD pathway is also a core ERAD route for degradation of non-native proteins in mammals. Central to this complex is the membranespanning HRD1 E3 ubiquitin ligase, which forms a stoichiometric complex with the membrane-anchored SEL1L (HRD3 in yeast). SEL1L is a type I membrane protein with a luminal tetratricopeptide repeat containing domain that is involved in binding and recognition of misfolded proteins (Gauss et al., 2006; Iida et al., 2011). 


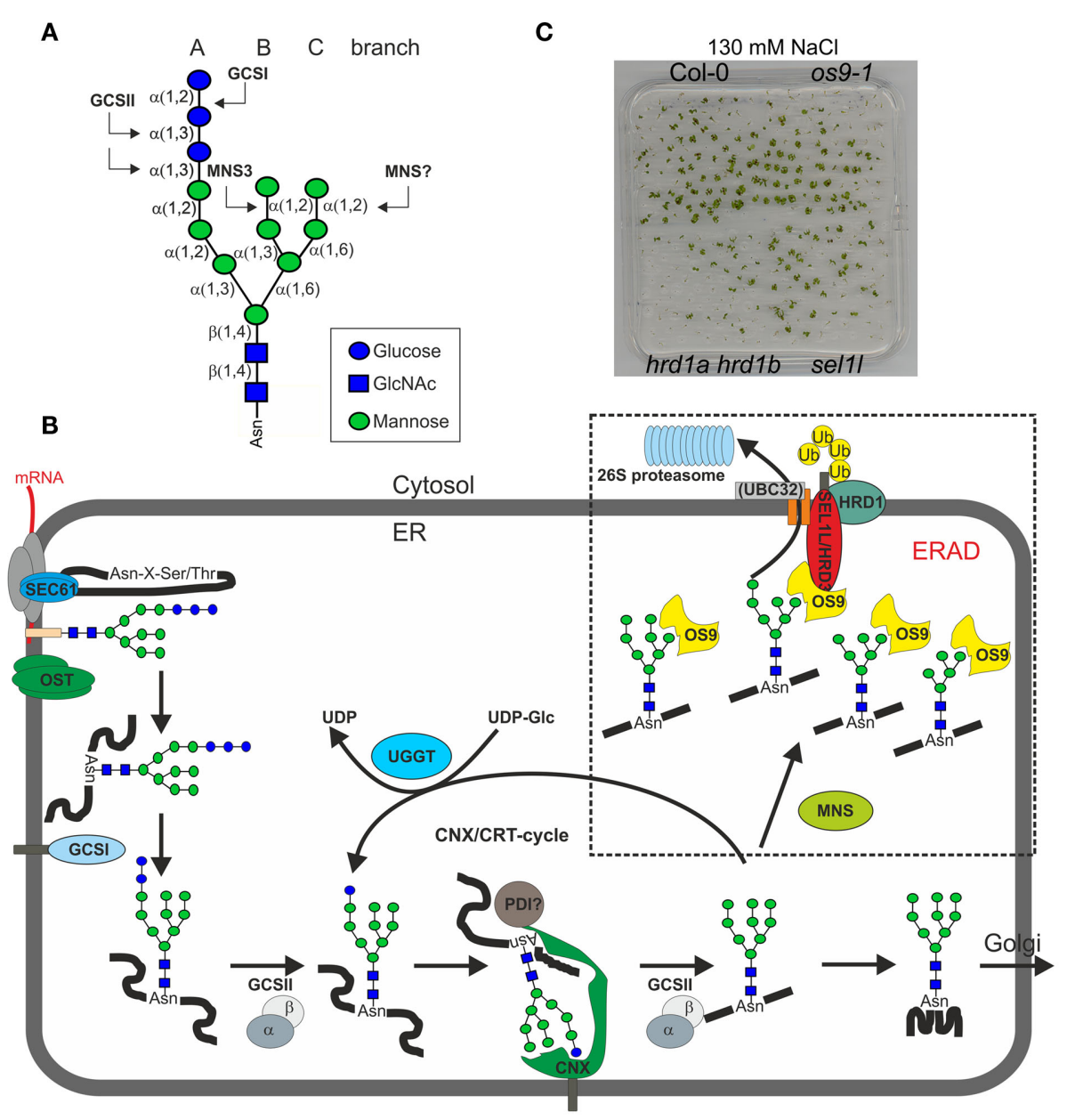

FIGURE 1 | (A) Schematic presentation of the $N$-linked glycan core structure. The enzymes (GCSI, GCSII, MNS3) involved in the first processing steps are indicated. Upon removal of the terminal $\alpha 1,2$-linked mannose by a so far unknown plant $\alpha$-mannosidase (MNS?) a free $\alpha 1,6$-mannose residue is exposed at the C-branch of the oligosaccharide, which presumably represents the glycan-specific degradation signal (Hong et al., 2009). (B) A proposed model for the role of $\mathrm{N}$-glycans in ER-quality control and ERAD in plants. Upon transfer of the oligosaccharide $\left(\mathrm{Glc}_{3} \mathrm{Man}_{9} \mathrm{GlcNAc}_{2}\right)$ from the lipid-linked precursor to asparagine residues of nascent polypeptides by the oligosaccharyltransferase complex (OST) the two terminal glucose residues are removed by $\alpha$-glucosidase I and II (GCSI, GCSII). The monoglucosylated $N$-glycan is a signal for recognition and binding by the lectins calreticulin (CRT - not shown here) and calnexin (CNX). Together with other folding catalysts like members of the protein disulfide isomerase (PDI) family, CNX/CRT promote folding. Properly folded glycoproteins are subsequently released from the CNX/CRT cycle and further processed by $\alpha$-mannosidases in the Golgi (Liebminger et al., 2009). Incompletely folded proteins can be re-glucosylated by
UDP-glucose:glycoprotein glucosyltransferase (UGGT) and are subjected to another round of CNX/CRT-mediated folding. Terminally misfolded glycoproteins are recognized by a poorly described mechanism, which involves the detection of a non-native protein conformation and mannose trimming by MNS proteins to generate a specific glycan code that is then recognized by OS9. Efficient disposal of glycoproteins requires the HRD1-SEL1L/HRD3-OS9 complex, which results in ubiquitylation (Ub) and subsequent degradation in the cytosol. UBC32 might participate in this complex or is part of a plant DOA10-like ERAD complex (not indicated here). As the precise glycan signal for degradation is still unknown, different oligomannosidic structures $\left(\mathrm{Man}_{5} \mathrm{GlcNAc}_{2}-\mathrm{Man}_{8} \mathrm{GlcNAc}_{2}\right)$ are shown. (C) Knockout of ERAD components results in reduced salt stress tolerance. However, the sel11, os 9 , and hrd1a hrd1b mutants display different degrees of sensitivity. HRD1-deficient plants display the most severe phenotype. Seeds were directly germinated on $0.5 \times \mathrm{MS}$ plates supplemented with $130 \mathrm{mM} \mathrm{NaCl}$ and grown for 16 days at $22^{\circ} \mathrm{C}$ with a 16-h-light photoperiod. The salt-sensitivity of se/1/ and os 9 has been described previously (Liu et al., 2011; Hüttner et al., 2012).
Another well-characterized component of the yeast HRD1 ERAD complex is the mannose-6-phosphate receptor homology (MRH) domain containing protein YOS9 (Bhamidipati et al., 2005; Kim et al., 2005; Szathmary et al., 2005). This luminal lectin interacts with HRD3 and together they perform proofreading of glycosylated ERAD substrates by recognition of a bipartite signal that is composed of a certain non-native protein conformation and the specific glycan signal (Hebert et al., 2010; Hosokawa et al., 2010). In the prevalent model, YOS9 or its mammalian counterparts OS9 and XTP3-B act as glycan-dependent sensors of client proteins and bind through their MRH-domain to an exposed a1,6-mannose residue at the C-branch of $N$-glycans on substrates destined for degradation (Figure 1A; Quan et al., 2008; Clerc et al., 2009; Hosokawa et al., 2009). In addition to the glycan-dependent 
binding it has also been shown that these MRH-domain proteins can bind to misfolded polypeptide segments and associate with the membrane-embedded ubiquitin ligase (Bhamidipati et al., 2005; Bernasconi et al., 2008; Christianson et al., 2008).

\section{ERAD OF GLYCOPROTEINS IN PLANTS}

In sharp contrast to mammals and yeast, glycan-dependent ERAD pathways are poorly described in plants (Vitale and Boston, 2008; Liu and Howell, 2010b; Ceriotti, 2011). A first evidence for ERretention and degradation of misfolded glycoproteins in plants has come from a study on an assembly defective form of phaseolin, which is the major storage protein of common bean and contains two N-glycosylation sites (Pedrazzini et al., 1997). The ER-tocytoplasm disposal route has also been described for a soluble GFP-form fused to the P-domain of maize calreticulin (Brandizzi et al., 2003). Another well-characterized ERAD substrate is the catalytic chain of the plant toxin ricin (RTA) from Ricinus communis. RTA is glycosylated and upon retrotranslocation to the cytosol, the protein is deglycosylated and eventually degraded in tobacco protoplasts (Di Cola et al., 2001). Proteasome inhibitors like clastolactacystin beta-lactone and MG132 stabilized RTA, but the mannose trimming inhibitor 1-deoxymannojirimycin had no effect on protein levels (Di Cola et al., 2005; Marshall et al., 2008) strongly indicating that this glycoprotein is degraded by a nonglycan-dependent ERAD pathway and thus does not represent a glycoprotein ERAD substrate. Interestingly, RTA and R. communis agglutinin (RCA), another ERAD substrate, are degraded in a ubiquitin-independent way and retrotranslocation and degradation are uncoupled. RTA and RCA disposal require the cytosolic ATPase CDC48 (Marshall et al., 2008), that acts as a molecular machine and provides the force to pull proteins out of the ER membrane to the proteasome. Expression of a dominant negative mutant of the Arabidopsis ATPase CDC48A, which is impaired in ATPase function (CDC48A QQ), causes also the accumulation of the non-glycosylated ERAD substrate MLO-1, which is a mutated form of the barley powdery mildew resistance $\mathrm{O}$ (MLO) protein (Müller et al., 2005). The integral membrane protein MLO-1 has a lesion in one of the cytoplasmic loops $\left(\right.$ ERAD $_{\mathrm{C}}$ substrate) and is therefore highly unstable when expressed in Arabidopsis. MLO1 is polyubiquitylated and degraded in a proteasome-dependent way and data from expression of MLO- 1 in yeast provide evidence that the disposal of misfolded MLO proteins is dependent on the HRD1 ubiquitin ligase complex.

Two mutated forms of the brassinosteroid receptor kinase BRASSINOSTEROID INSENSITIVE 1 (BRI1) have been classified as glycoprotein ERAD substrates (Hong et al., 2008, 2009). BRI1 is a plasma membrane protein that is subjected to receptor internalization and eventually degraded in the vacuole (Russinova et al., 2004). BRI1 contains a single-pass transmembrane domain and $14 \mathrm{~N}$-glycosylation sites in its N-terminal extracellular brassinosteroid binding domain ( $\mathrm{Li}$ and Chory, 1997). The two mutated receptor variants BRI1-5 (C69Y mutation, ERAD substrate) and BRI1-9 (S662F mutation, ERAD $\mathrm{L}_{\mathrm{L}}$ substrate) display a subtle change in their structural conformation that retains them in the ER by different protein quality control mechanisms (Jin et al., 2007, 2009; Hong et al., 2008). Pharmacological inhibition of mannose trimming using the highly specific class I $\alpha$-mannosidase inhibitor kifunensine stabilizes both BRI1-5 and BRI1-9, resulting in their partial leakage to the plasma membrane and suppression of the severe growth phenotype of bri1-5 and bri1-9 mutants (Hong et al., 2008, 2009). In addition, the bri1-5 and bri1-9 growth defects are also rescued by the alg12 mutant, which lacks the specific mannosyltransferase that transfers the $\alpha 1,6$-mannose to the C-branch during the assembly of the oligosaccharide precursor (Hong et al., 2009). These findings are hallmarks of glycan-dependent ERAD processes and reveal that recognition of a defined mannose residue plays also a crucial role for the degradation of aberrant glycoproteins in plants.

Recently, the first members of the Arabidopsis ERAD complex have been discovered (Liu et al., 2011; Su et al., 2011). Mutants deficient in the homologs of the membrane-bound cargo receptor SEL1L/HRD3 and the E3 ubiquitin ligase HRD1 can suppress the dwarf phenotype of bril-5 and bril-9 plants. selll/hrd3 mutants accumulate BRI1-5 and BRI1-9 proteins and analysis of their glycosylation status revealed the presence of processed endoglycosidase $\mathrm{H}$-insensitive $\mathrm{N}$-glycans on a small portion of the mutant BRI1 variants. These data suggest transport of functional BRI1 variants to the plasma membrane resulting in the rescue of the dwarf phenotypes of bri1-5 and bri1-9. A similar phenotypic suppression of the bril-9 phenotype and BRI1-9 accumulation was observed for a mutant (hrdla hrd1b) with T-DNA insertions in both copies of the Arabidopsis HRD1 homolog (Su et al., 2011). Moreover, stabilization of the non-glycosylated $E_{R A D}$ substrate MLO-1 was detected in sel1l/hrd3 plants (Liu et al., 2011) indicating that the HRD1-SEL1L/HRD3 complex is involved in degradation of glycosylated as well as non-glycosylated proteins (Table 1).

In another recent study the putative Arabidopsis homolog of YOS9 termed OS9 was identified and characterized with respect to its role in degradation of misfolded glycoproteins (Hüttner et al.,

Table 1 | List of identified ERAD proteins from $A$. thaliana.

\begin{tabular}{lllll}
\hline Protein name & Arabidopsis name & Accession number & ERAD substrate & Reference \\
\hline SEL1L/HRD3 & HRD3A ${ }^{2}$, EBS5 & At1g18260 & BRI1-5, BRI1-9, MLO-1 & Su et al. (2011), Liu et al. (2011) \\
HRD1/HRD1 & HRD1A, HRD1B & At3g16090, At1g65040 & BRI1-9 & Su et al. (2011) \\
OS9, XTP3-B/YOS9 & OS9 & At5g35080 & BRI1-5, BRI1-9 & Hüttner et al. (2012) \\
UBE2J1/UBC6 & UBC32 & At3g17000 & BRI1-5, BRI1-9, MLO-12 & Cui et al. (2012) \\
\hline
\end{tabular}

'The names of the mammalian and yeast homologs are given.

${ }^{2}$ There is another SEL1L homolog in the A. thaliana genome (HRD3B), which is a pseudogene and does not play any role in ERAD (Liu et al., 2011; Su et al., 2011). 
2012). Consistent with a role in ERAD, the os 9 mutant suppresses the bril-5 and bri1-9 phenotypes and a chimeric protein consisting of the Arabidopsis OS9 protein fused to the C-terminal region of Saccharomyces cerevisiae YOS9 was able to complement the protein degradation defect of the $\Delta$ yos 9 yeast mutant. Coimmunoprecipitation analysis of transiently expressed proteins revealed that OS9 interacts with SEL1L/HRD3 as well as with BRI1-5 and BRI1-9, strongly indicating that this plant MRHdomain containing protein has a similar substrate proofreading function with recognition of a distinct glycan signal on ERAD substrates. Together these findings demonstrate that a conserved HRD1-SEL1L/HRD3-OS9 pathway for the degradation of terminally misfolded proteins exists in the ER of plants. Despite this recent progress, the nature of the glycan signal and the process that leads to its generation on aberrant proteins is still unknown. The aforementioned results for increased BRI1-5 and BRI1-9 protein levels as a result of kifunensine treatment and alg12-mediated suppression of the bri1-5/bri1-9 phenotype highlight that mannose trimming orchestrated by $\alpha$-mannosidases is essential for degradation of these misfolded BRI1 receptors. The Arabidopsis class I $\alpha$-mannosidase family consists of five members (MNS1 to MNS5; Liebminger et al., 2009). The MNS proteins remove one to four mannose residues from the oligomannosidic core $\mathrm{N}$-glycan and thus at least one of them acts upstream of OS9 to generate the defined glycan signal, presumably a Man5-Man8 structure containing a terminal $\alpha 1,6$-linked mannose at the C-branch that targets anomalous proteins like BRI1-5/BRI1-9 for degradation through the ERAD pathway (Figures 1A,B).

In contrast to that, the downstream processes of BRI1-5 and BRI1-9 disposal during ERAD are less clear. The proteasomedependent degradation of the mutated BRI1 variants was only shown for ectopically expressed BRI1-9-GFP (Hong et al., 2009). Endogenous BRI1-5 on the other hand did not accumulate in the presence of the proteasome inhibitor MG132 (Hong et al., 2008). In addition, the distinct role of HRD1 is unclear and retrotranslocation as well as ubiquitylation of BRI1-5 and BRI1-9 remains to be shown. It cannot be excluded that degradation of these two proteins is not mediated by the conventional ERAD pathway as described for yeast and metazoans, but by an alternative mechanism that involves components of the core ERAD machinery (like OS9, SEL1L/HRD3) and leads to degradation via other routes like the delivery to the vacuole (Pimpl et al., 2006; Foresti et al., 2008). In mammalian cells an interplay between proteasomal and lysosomal degradation pathways has been described for an ERAD substrate (Chiang et al., 2012) and ERAD competes with ER export pathways (Kincaid and Cooper, 2007; Kawaguchi et al., 2010), which affects the fate and finally the site of protein disposal in the cell.

\section{IS THERE A REGULATORY FUNCTION OF THE PLANT ERAD COMPLEX?}

Apart from degradation of aberrant proteins the ERAD machinery plays also a major role in physiological regulation of protein turnover and constitutive degradation of processed polypeptide fragments in yeast and mammalian cells (Kikkert et al., 2004; Chen et al., 2011; Jaenicke et al., 2011). The identification of plant ERAD components allows now to address the question whether ERAD in plants has in addition to the removal of non-native proteins also a similar function in regulation of protein turnover. Remarkably, none of the three Arabidopsis ERAD mutants (selll/hrd3, hrd1a $h r d 1 b, o s 9)$ displays an obvious phenotype under normal growth conditions. However, all three mutants are less tolerant toward salt stress, with $h r d 1 a$ hrd1b being more affected than sel1l/hrd3 and os9 (Liu et al., 2011; Hüttner et al., 2012; Figure 1C). In regard to that, all three ERAD components are upregulated by chemicals like tunicamycin that result in the accumulation of misfolded proteins and induce the unfolded protein response (UPR; Martínez and Chrispeels, 2003; Kamauchi et al., 2005; Nagashima et al., 2011; Su et al., 2011; Hüttner et al., 2012). Current models predict a relationship between salt stress response and UPR induction. High salt concentrations may result in the accumulation of misfolded or unfolded proteins in the ER leading to the activation of salt stress responsive and UPR pathway genes (Liu et al., 2007, 2011; Che et al., 2010; Liu and Howell, 2010a). Under such adverse environmental conditions, the ERAD complex could alleviate ER stress by removal of misfolded proteins, while in the absence of a functional ERAD pathway the growth/survival of plants is impaired (Figure 1C; Liu et al., 2011; Hüttner et al., 2012).

In a recent study, the ubiquitin conjugating enzyme UBC32 has been identified as another component involved in ERAD (Cui et al., 2012). UBC32 deficiency resulted in the accumulation of BRI1-5/BRI1-9 proteins and subsequent suppression of the bri15 and bri1-9 phenotypes. UBC32, which is homologous to yeast UBC6, is very likely a component of the plant DOA10 complex (Cui et al., 2012). Interestingly, the stabilization of BRI1 variants and suppression of their growth defect indicates overlapping client proteins with the HRD1-SEL1L/HRD3-OS9 complex. However, there is a major difference with respect to the salt stress response as the $u b c 32$ mutants are more tolerant to salt stress and overexpression of UBC32 results in increased salt-sensitivity. These findings suggest that UBC32 is either a negative regulator of the pathway or has additional unknown functions that are unrelated to the degradation of misfolded proteins under ER stress situations. Further investigation of the pathways and complexes are required to solve this discrepancy.

Based on the finding that $u b c 32$ seedlings are more sensitive to brassinosteroids in a hypocotyl elongation assay it has been proposed that there is a link between ERAD, the salt stress response, and brassinosteroid signaling due to a direct effect of UBC32 on BRI1 (Cui et al., 2012). However, no evidence was provided that wild-type BRI1 protein levels are altered in $u b c 32$ or UBC32 overexpression lines. Other studies have shown that native BRI1 is not subjected to ERAD and does not interact with ERAD components (Hong et al., 2008, 2009; Su et al., 2011; Hüttner et al., 2012). Consequently, it might in fact be that UBC32 is involved in processes that indirectly affect brassinosteroid signaling. In this context we would like to emphasize that the identified ERAD substrates BRI1-5 and BRI1-9 are artificial clients, which are valuable tools to examine the plant ERAD pathway and its components, but do not provide any direct insights into the function of the ERAD complex under physiological conditions. To investigate the regulatory function of the ERAD complex it is essential to identify endogenous target proteins that are degraded via this pathway. A recent proteomics approach in yeast has discovered 85 proteins 
that were significantly more abundant in a mutant with a deficiency in HRD1 (Jaenicke et al., 2011) indicating that the core ERAD pathway plays an important role for protein turnover under non-ER stress conditions.

\section{CHALLENGES AND PERSPECTIVES}

The main shortcoming of ERAD research in plants is the lack of suitable client proteins to investigate individual steps and discover novel aspects of the pathway. For example, no luminal glycoprotein ERAD substrate has been identified so far and it is still not entirely clear how ER-retained glycoprotein ERAD substrates are degraded as ubiquitylation and translocation to the cytoplasm remains to be shown. Binding of SEL1L/HRD3 and OS9 to the misfolded BRI1-5 and BRI1-9 proteins suggests that these ERAD components have a direct role in targeting of substrates for disposal (Su et al., 2011; Hüttner et al., 2012). However, apart from this proposed direct

\section{REFERENCES}

Aebi, M., Bernasconi, R., Clerc, S., and Molinari, M. (2010). N-glycan structures: recognition and processing in the ER. Trends Biochem. Sci. 35, 74-82.

Bernasconi, R., Galli, C., Calanca, V., Nakajima, T., and Molinari, M. (2010). Stringent requirement for HRD1, SEL1L, and OS-9/XTP3-B for disposal of ERAD-LS substrates. J. Cell Biol. 188, 223-235.

Bernasconi, R., Pertel, T., Luban, J., and Molinari, M. (2008). A dual task for the Xbpl-responsive OS-9 variants in the mammalian endoplasmic reticulum: inhibiting secretion of misfolded protein conformers and enhancing their disposal. J. Biol. Chem. 283, 16446-16454.

Bhamidipati, A., Denic, V., Quan, E. M., and Weissman, J. S. (2005). Exploration of the topological requirements of ERAD identifies Yos9p as a lectin sensor of misfolded glycoproteins in the ER lumen. Mol. Cell 19, 741-751.

Brandizzi, F., Hanton, S., DaSilva, L., Boevink, P., Evans, D., Oparka, K., Denecke, J., and Hawes, C. (2003). ER quality control can lead to retrograde transport from the ER lumen to the cytosol and the nucleoplasm in plants. Plant J. 34, 269-281.

Caramelo, J., and Parodi, A. (2008). Getting in and out from calnexin/calreticulin cycles. J. Biol. Chem. 283, 10221-10225.

Ceriotti, A. (2011). Waste disposal in the endoplasmic reticulum, ROS production and plant salt stress response. Cell Res. 21, 555-557.

Che, P., Bussell, J. D., Zhou, W., Estavillo, G. M., Pogson, B. J., and Smith, S. M. (2010). Signaling from the endoplasmic reticulum activates brassinosteroid signaling and promotes acclimation to stress in Arabidopsis. Sci. Signal. 3, ra69.

Chen, X., Tukachinsky, H., Huang, C. H., Jao, C., Chu, Y. R., Tang, H. Y., Mueller, B., Schulman, S., Rapoport, T. A., and Salic, A. (2011). Processing and turnover of the Hedgehog protein in the endoplasmic reticulum. $J$. Cell Biol. 192, 825-838.

Chiang, W. C., Messah, C., and Lin, J. H. (2012). IRE1 directs proteasomal and lysosomal degradation of misfolded rhodopsin. Mol. Biol. Cell 23, 758-770.

Christianson, J. C., Olzmann, J. A., Shaler, T. A., Sowa, M. E., Bennett, E. J., Richter, C. M., Tyler, R. E., Greenblatt, E. J., Harper, J. W., and Kopito, R. R. (2012). Defining human ERAD networks through an integrative mapping strategy. Nat. Cell Biol. 14, 93-105.

Christianson, J. C., Shaler, T. A., Tyler, R. E., and Kopito, R. R. (2008). OS-9 and GRP94 deliver mutant alpha1antitrypsin to the Hrd1-SEL1L ubiquitin ligase complex for ERAD. Nat. Cell Biol. 10, 272-282.

Clerc, S., Hirsch, C., Oggier, D. M., Deprez, P., Jakob, C., Sommer, T., and Aebi, M. (2009). Html protein generates the $\mathrm{N}$-glycan signal for glycoprotein degradation in the endoplasmic reticulum. J. Cell Biol. 184, 159-172.

Cui, F., Liu, L., Zhao, Q., Zhang, Z., Li, Q., Lin, B., Wu, Y., Tang, S., and Xie, Q. (2012). Arabidopsis ubiquitin conjugase UBC32 is an ERAD component that functions in brassinosteroid-mediated salt stress tolerance. Plant Cell 24, 233-244.

Denic, V., Quan, E. M., and Weissman, J. S. (2006). A luminal surveillance complex that selects misfolded glycoproteins for ER-associated degradation. Cell 126, 349-359.

Di Cola, A., Frigerio, L., Lord, J. M., Ceriotti, A., and Roberts, L. M.

effect we cannot rule out that BRI1-5/BRI1-9 protein accumulation or ER-retention are altered by an unknown mechanism in the absence of a functional ERAD pathway. The nature of the glycan signal and its generation are also still poorly understood and no endogenous glycoprotein substrates are known. In conclusion, the recent identification of plant ERAD components is a major breakthrough since it enables now the concerted search for interacting proteins and new ERAD substrates that undergo glycan-mediated degradation in the ER. Moreover, the controversial data regarding the salt stress tolerance suggests a complex regulation and interaction of ERAD with other ER stress-induced pathways, like the UPR, that have to be discovered in the future.

\section{ACKNOWLEDGMENTS}

This work was supported by a grant from Austrian Science Fund (FWF): P20817-B12.

(2001). Ricin A chain without its partner B chain is degraded after retrotranslocation from the endoplasmic reticulum to the cytosol in plant cells. Proc. Natl. Acad. Sci. U.S.A. 98, 14726-14731.

Di Cola, A., Frigerio, L., Lord, J. M., Roberts, L., and Ceriotti, A. (2005). Endoplasmic reticulumassociated degradation of ricin A chain has unique and plantspecific features. Plant Physiol. 137, 287-296.

Foresti, O., De Marchis, F., de Virgilio, M., Klein, E. M., Arcioni, S., Bellucci, M., and Vitale, A. (2008). Protein domains involved in assembly in the endoplasmic reticulum promote vacuolar delivery when fused to secretory GFP, indicating a protein quality control pathway for degradation in the plant vacuole. Mol. Plant 1, 1067-1076

Gauss, R., Jarosch, E., Sommer, T., and Hirsch, C. (2006). A complex of Yos9p and the HRD ligase integrates endoplasmic reticulum quality control into the degradation machinery. Nat. Cell Biol. 8, 849-854.

Hebert, D. N., Bernasconi, R., and Molinari, M. (2010). ERAD substrates: which way out? Semin. Cell Dev. Biol. 21, 526-532.

Hegde, R. S., and Ploegh, H. L. (2010). Quality and quantity control at the endoplasmic reticulum. Curr. Opin. Cell Biol. 22, 437-446.

Helenius, A., and Aebi, M. (2004). Roles of N-linked glycans in the endoplasmic reticulum. Annu. Rev. Biochem. 73, 1019-1049.

Hong, Z., Jin, H., Fitchette, A.-C., Xia, Y., Monk, A. M., Faye, L., and Li, J. (2009). Mutations of an alphal,6 mannosyltransferase inhibit endoplasmic reticulum-associated degradation of defective brassinosteroid receptors in Arabidopsis. Plant Cell 21, 3792-3802.
Hong, Z., Jin, H., Tzfira, T., and Li, J. (2008). Multiple mechanismmediated retention of a defective brassinosteroid receptor in the endoplasmic reticulum of Arabidopsis. Plant Cell 20, 3418-3429.

Hosokawa, N., Kamiya, Y., Kamiya, D., Kato, K., and Nagata, K. (2009). Human OS-9, a lectin required for glycoprotein endoplasmic reticulum-associated degradation, recognizes mannose-trimmed N-glycans. J. Biol. Chem. 284, 17061-17068.

Hosokawa, N., Kamiya, Y., and Kato, K. (2010). The role of MRH domaincontaining lectins in ERAD. Glycobiology 20, 651-660.

Hüttner, S., Veit, C., Schoberer, J., Grass, J., and Strasser, R. (2012). Unraveling the function of Arabidopsis thaliana OS9 in the endoplasmic reticulum-associated degradation of glycoproteins. Plant Mol. Biol. doi:10.1007/s11103-012-9891-4

Iida, Y., Fujimori, T., Okawa, K., Nagata, K., Wada, I., and Hosokawa, N. (2011). SEL1L protein critically determines the stability of the HRD1-SEL1L endoplasmic reticulum-associated degradation (ERAD) complex to optimize the degradation kinetics of ERAD substrates. J. Biol. Chem. 286, 16929-16939.

Jaenicke, L. A., Brendebach, H., Selbach, M., and Hirsch, C. (2011). Yos9p assists in the degradation of certain nonglycosylated proteins from the endoplasmic reticulum. Mol. Biol. Cell 22, 2937-2945.

Jin, H., Hong, Z., Su, W., and Li, J. (2009). A plant-specific calreticulin is a key retention factor for a defective brassinosteroid receptor in the endoplasmic reticulum. Proc. Natl. Acad. Sci. U.S.A. 106, 13612-13617. 
Jin, H., Yan, Z., Nam, K., and Li, J. (2007). Allele-specific suppression of a defective brassinosteroid receptor reveals a physiological role of UGGT in ER quality control. Mol. Cell 26, 821-830.

Kamauchi, S., Nakatani, H., Nakano, C., and Urade, R. (2005). Gene expression in response to endoplasmic reticulum stress in Arabidopsis thaliana. FEBS J. 272, 3461-3476.

Kawaguchi, S., Hsu, C.-L., and Ng, D. T. W. (2010). Interplay of substrate retention and export signals in endoplasmic reticulum quality control. PLoS ONE 5, el5532. doi:10.1371/journal.pone.0015532

Kikkert, M., Doolman, R., Dai, M., Avner, R., Hassink, G., van Voorden, S., Thanedar, S., Roitelman, J., Chau, V., and Wiertz, E. (2004). Human HRD1 is an E3 ubiquitin ligase involved in degradation of proteins from the endoplasmic reticulum. $J$. Biol. Chem. 279, 3525-3534.

Kim, W., Spear, E. D., and Ng, D. T. W. (2005). Yos9p detects and targets misfolded glycoproteins for ERassociated degradation. Mol. Cell 19, 753-764.

Kincaid, M. M., and Cooper, A. A. (2007). Misfolded proteins traffic from the endoplasmic reticulum (ER) due to ER export signals. Mol. Biol. Cell 18, 455-463.

Li, J., and Chory, J. (1997). A putative leucine-rich repeat receptor kinase involved in brassinosteroid signal transduction. Cell 90, 929-938.

Liebminger, E., Hüttner, S., Vavra, U., Fischl, R., Schoberer, J., Grass, J., Blaukopf, C., Seifert, G. J., Altmann, F., Mach, L., and Strasser, R. (2009). Class I alpha-mannosidases are required for $\mathrm{N}$-glycan processing and root development in Arabidopsis thaliana. Plant Cell 21, 3850-3867.
Liu, J.-X., Srivastava, R., Che, P., and Howell, S. H. (2007). Salt stress responses in Arabidopsis utilize a signal transduction pathway related to endoplasmic reticulum stress signaling. Plant J. 51, 897-909.

Liu, J.-X., and Howell, S. H. (2010a). bZIP28 and NF-Y transcription factors are activated by ER stress and assemble into a transcriptional complex to regulate stress response genes in Arabidopsis. Plant Cell 22, 782-796.

Liu, J.-X., and Howell, S. H. (2010b). Endoplasmic reticulum protein quality control and its relationship to environmental stress responses in plants. Plant Cell 22, 2930-2942.

Liu, L., Cui, F., Li, Q., Yin, B., Zhang, H., Lin, B., Wu, Y., Xia, R., Tang, S., and Xie, Q. (2011). The endoplasmic reticulum-associated degradation is necessary for plant salt tolerance. Cell Res. 21, 957-969.

Marshall, R. S., Jolliffe, N. A., Ceriotti, A., Snowden, C. J., Lord, J. M., Frigerio, L., and Roberts, L. M. (2008). The role of CDC48 in the retrotranslocation of non-ubiquitinated toxin substrates in plant cells. J. Biol. Chem. 283, 15869-15877.

Martínez, I., and Chrispeels, M. (2003). Genomic analysis of the unfolded protein response in Arabidopsis shows its connection to important cellular processes. Plant Cell 15, 561-576.

Müller, J., Piffanelli, P., Devoto, A., Miklis, M., Elliott, C., Ortmann, B., Schulze-Lefert, P., and Panstruga, R. (2005). Conserved ERAD-like quality control of a plant polytopic membrane protein. Plant Cell 17, 149-163.

Nagashima, Y., Mishiba, K., Suzuki, E., Shimada, Y., Iwata, Y., and Koizumi, N. (2011). Arabidopsis
IRE1 catalyses unconventional splicing of bZIP60 mRNA to produce the active transcription factor. Sci. Rep. 1, 29.

Pedrazzini, E., Giovinazzo, G., Bielli, A., de Virgilio, M., Frigerio, L., Pesca, M., Faoro, F., Bollini, R., Ceriotti, A., and Vitale, A. (1997). Protein quality control along the route to the plant vacuole. Plant Cell 9, 1869-1880.

Pimpl, P., Taylor, J. P., Snowden, C., Hillmer, S., Robinson, D. G., and Denecke, J. (2006). Golgi-mediated vacuolar sorting of the endoplasmic reticulum chaperone BiP may play an active role in quality control within the secretory pathway. Plant Cell 18, 198-211.

Quan, E., Kamiya, Y., Kamiya, D., Denic, V., Weibezahn, J., Kato, K., and Weissman, J. S. (2008). Defining the glycan destruction signal for endoplasmic reticulum-associated degradation. Mol. Cell 32, 870-877.

Russinova, E., Borst, J.-W., Kwaaitaal, M., Caño-Delgado, A., Yin, Y., Chory, J., and de Vries, S. C. (2004). Heterodimerization and endocytosis of Arabidopsis brassinosteroid receptors BRI1 and AtSERK3 (BAK1). Plant Cell 16, 3216-3229.

Sato, B. K., Schulz, D., Do, P. H., and Hampton, R. Y. (2009). Misfolded membrane proteins are specifically recognized by the transmembrane domain of the Hrdlp ubiquitin ligase. Mol. Cell 34, 212-222.

Smith, M. H., Ploegh, H. L., and Weissman, J. S. (2011). Road to ruin: targeting proteins for degradation in the endoplasmic reticulum. Science 334, 1086-1090.

Su, W., Liu, Y., Xia, Y., Hong, Z., and Li, J. (2011). Conserved endoplasmic reticulum-associated degradation system to eliminate mutated receptor-like kinases in Arabidopsis. Proc. Natl. Acad. Sci. U.S.A. 108, 870-875.

Szathmary, R., Bielmann, R., Nita-Lazar, M., Burda, P., and Jakob, C. A. (2005). Yos9 protein is essential for degradation of misfolded glycoproteins and may function as lectin in ERAD. Mol. Cell 19, 765-775.

Vashist, S., and Ng, D. T. W. (2004). Misfolded proteins are sorted by a sequential checkpoint mechanism of ER quality control. J. Cell Biol. 165, 41-52.

Vitale, A., and Boston, R. S. (2008). Endoplasmic reticulum quality control and the unfolded protein response: insights from plants. Traffic 9, 1581-1588.

Conflict of Interest Statement: The authors declare that the research was conducted in the absence of any commercial or financial relationships that could be construed as a potential conflict of interest.

Received: 12 March 2012; accepted: 21 March 2012; published online: 05 April 2012.

Citation: Hüttner $S$ and Strasser $R$ (2012) Endoplasmic reticulumassociated degradation of glycoproteins in plants. Front. Plant Sci. 3:67. doi: 10.3389/fpls.2012.00067

This article was submitted to Frontiers in Plant Cell Biology, a specialty of Frontiers in Plant Science.

Copyright (ㄷ) 2012 Hüttner and Strasser. This is an open-access article distributed under the terms of the Creative Commons Attribution Non Commercial License, which permits non-commercial use, distribution, and reproduction in other forums, provided the original authors and source are credited. 